\title{
Stationary bootstrapping for structural break tests for a heterogeneous autoregressive model
}

\author{
Eunju Hwang $^{a}$, Dong Wan Shin ${ }^{1, b}$ \\ ${ }^{a}$ Department of Applied Statistics, Gachon University, Korea; \\ ${ }^{b}$ Department of Statistics, Ewha Womans University, Korea
}

\begin{abstract}
We consider an infinite-order long-memory heterogeneous autoregressive (HAR) model, which is motivated by a long-memory property of realized volatilities (RVs), as an extension of the finite order HAR-RV model. We develop bootstrap tests for structural mean or variance changes in the infinite-order HAR model via stationary bootstrapping. A functional central limit theorem is proved for stationary bootstrap sample, which enables us to develop stationary bootstrap cumulative sum (CUSUM) tests: a bootstrap test for mean break and a bootstrap test for variance break. Consistencies of the bootstrap null distributions of the CUSUM tests are proved. Consistencies of the bootstrap CUSUM tests are also proved under alternative hypotheses of mean or variance changes. A Monte-Carlo simulation shows that stationary bootstrapping improves the sizes of existing tests.
\end{abstract}

Keywords: heterogeneous autoregressive $(\infty)$ model, stationary bootstrap, structural changes, CUSUM test

\section{Introduction}

Corsi (2009) and Hwang and Shin (2014) recently proposed autoregressive models called heterogeneous autoregressions (HAR) of realized volatility (RV) to address the long-memory properties of financial market volatilities. Corsi $(2004,2009)$ proposed an additive cascade model having three volatility components defined over three different time periods, called the HAR(3) model. The HAR(3) model has been shown to successively achieve the purpose of reproducing the main empirical features of financial return volatilities such as long memory, fat tails and self-similarity. However, as noted by Corsi (2009), the HAR(3) model has a short memory with a exponentially decreasing autocorrelation function (ACF) because it can be expressed as a stationary AR(22) model. Hwang and Shin (2014) proposed a genuine long-memory HAR model with algebraically decreasing ACF, an infinite-order HAR $(\infty)$ model, as an extension of the Corsi (2009)'s HAR(3) model. They characterized stationary conditions for the model, provided some probability theories and statistical methods in terms of consistency and limited the normality of the ordinary least squares estimator (OLSE) and forecasting.

Long memory of realized volatility is occasionally accompanied by structural changes. The problem of testing for structural changes has been a most important issue in time series regression or dynamic economic models. For this purpose, cumulative sum (CUSUM) tests have been widely used because the change-points are not known. See Brown et al. (1975), Ploberger and Krämer (1986,

\footnotetext{
${ }^{1}$ Corresponding author: Department of Statistics, Ewha Womans University, 52 Ewhayeodae-gil, Seodaemun-gu, Seoul 03760, Korea. E-mail: shindw@ewha.ac.kr
}

Published 31 July 2017 / journal homepage: http://csam.or.kr

(C) 2017 The Korean Statistical Society, and Korean International Statistical Society. All rights reserved. 
1990, 1992), Qu and Perron (2007), and Deng and Perron (2008) for the CUSUM(-SQ) tests. For more efficient versions of the CUSUM tests, we refer to Xu $(2013,2015)$, who focused on volatility and mean change tests and proposed powerful and robust alternatives. In the long-memory HAR models, Hwang and Shin $(2013,2015)$ and Lee (2014) studied CUSUM tests for mean or variance breaks. In particular, Lee (2014) established a functional central limit theorem (FCLT), by proving that the $\operatorname{HAR}(\infty)$ process is a near epoch dependent (NED) process, which has been applied to construct a CUSUM test for mean stability and a CUSUM test for variance stability.

All the break tests for the HAR model except the CUSUMSQ test of Hwang and Shin (2015) have undesirable size distortions. The aim of this paper is to develop bootstrap tests for mean or variance changes in the HAR $(\infty)$ model, which remedy the size distortion problem. Based on the result of Lee (2014), we establish a bootstrap FCLT. Block bootstrapping methods are more well-suited than identically distributed (iid) bootstrapping because realized volatilities have long memories. Among the various block bootstrapping methods used, we consider the stationary bootstrapping (SB) of Politis and Romano (1994). The SB is one of the most widely adopted block bootstrapping methods for the dependent samples and is characterized by geometrically distributed random block lengths.

The partial sum process of the SB sample is shown to converge to the standard Brownian motion that enables us to construct SB CUSUM tests: a bootstrap mean break test and a bootstrap variance break test. Asymptotic critical values can be obtained from the stationary bootstrap distribution of the CUSUM tests. Consistencies of the null bootstrapping distributions of the CUSUM tests are proved. Consistencies of the bootstrapping CUSUM tests are also proved under alternative hypotheses of mean or variance breaks.

A Monte-Carlo experiment is conducted to show that SB significantly improves the sizes of the CUSUM tests of Lee (2014) for mean break and for variance break, which are badly sized in a finite sample. It also shows some improvement of the CUSUM test of Hwang and Shin (2013) for the mean break. The size improvement is achieved without power loss.

The remaining of the paper is organized as follows. The HAR models are described and Section 2 presents the existing results Section 3 discusses the main results, including the SB functional central theorem and the bootstrap CUSUM tests. Section 4 deals with the Monte-Carlo study and Section 5 gives the concluding remarks. The Appendix provides the proofs.

\section{Existing theories and methods}

\subsection{Heterogeneous autoregressive models}

First, we describe the $3^{\text {rd }}$ order HAR(3) model of Corsi (2009) defined by

$$
Y_{t}=\beta_{0}+\sum_{j=1}^{p} \beta_{j} Y_{t, h_{j}}+a_{t},
$$

where $p=3, a_{t}$ is a sequence of regression error,

$$
Y_{t, h_{j}}=\frac{1}{h_{j}}\left(Y_{t-1}+Y_{t-2}+\cdots+Y_{t-h_{j}}\right), \quad j=1,2, \ldots,
$$

$h_{1}=1, h_{2}=5$ and $h_{3}=22$. Note that $Y_{t, h_{j}}, j=2,3$, are weekly and monthly moving averages, respectively. This model captures long-memory in a parameter-parsimonious way by considering the moving averages. However, this model is theoretically a short-memory $\operatorname{AR}(22)$ model having exponentially decreasing ACF and is not a genuine long-memory model. 
As an extension of Corsi's model (2.1), Hwang and Shin (2014) proposed an infinite-order genuine long-memory $\operatorname{HAR}(\infty)$ model having an algebraically decreasing $\mathrm{ACF}$, defined by

$$
Y_{t}=\beta_{0}+\beta_{1} Y_{t, h_{1}}+\beta_{2} Y_{t, h_{2}}+\cdots+\epsilon_{t},
$$

where $Y_{t, h_{j}}$ is as in (2.2), $\left\{\beta_{j}: j=0,1,2, \ldots\right\}$ is a sequence of real numbers tending to $0,\left\{h_{j}: j=\right.$ $1,2, \ldots\}$ is a given sequence of positive integers increasing to $\infty$, and $\left\{\epsilon_{t}\right\}$ is a sequence of iid random variables with mean zero and variance $E\left[\epsilon_{t}^{2}\right]=\sigma_{\epsilon}^{2}$.

Here, we review the discussion of Hwang and Shin (2014) for the HAR( $\infty)$ process $Y_{t}$ in $(2.3)$ and adopt their assumptions on the $\operatorname{HAR}(\infty)$. The HAR $(\infty)$ process $Y_{t}$ in $(2.3)$ can be written as an $\operatorname{AR}(\infty)$ process:

$$
Y_{t}=\beta_{0}+\sum_{j=1}^{\infty} \frac{\beta_{j}}{h_{j}}\left(Y_{t-1}+Y_{t-2}+\cdots+Y_{t-h_{j}}\right)+\epsilon_{t}=\beta_{0}+\sum_{j=1}^{\infty} \alpha_{j}\left(\sum_{k=h_{j-1}+1}^{h_{j}} Y_{t-k}\right)+\epsilon_{t}=\beta_{0}+\sum_{i=1}^{\infty} \phi_{i} Y_{t-i}+\epsilon_{t}
$$

with $\alpha_{j}=\sum_{k=j}^{\infty} \beta_{k} / h_{k}$ for $j=1,2, \ldots ; \phi_{1}=\alpha_{1}, \phi_{h_{j}+r}=\alpha_{j+1}$ for $r=1,2, \ldots, h_{j+1}-h_{j}$ and $j=1,2, \ldots$; and $h_{0}=0$. We need the following conditions for the stationarity of $Y_{t}$ :

(A1) The coefficients $\beta_{j}$ in (2.3) satisfy $\sum_{j=1}^{\infty}\left|\beta_{j}\right|<\infty$, and $A(z) \neq 0$ for $|z| \leq 1$ where the polynomial

$$
A(z)=1-\sum_{j=1}^{\infty} \alpha_{j} f_{j}(z)=1-\sum_{i=1}^{\infty} \phi_{i} z^{i}
$$

with $f_{j}(z)=\sum_{k=h_{j-1}+1}^{h_{j}} z^{k}$ for $j=1,2, \ldots$

We refer to Remarks 1 and 2 of Hwang and Shin (2014) for a necessary and sufficient condition of (A1) and for the absolutely summability of $\phi_{i}$ and $\alpha_{j}$. Proposition 1 characterizes stationarity of $Y_{t}$, which is given by Hwang and Shin (2014).

Proposition 1. Assume condition (Al). Then $Y_{t}$ is stationary and has a one-sided infinite movingaverage representation $Y_{t}=\mu+\sum_{k=0}^{\infty} \xi_{k} \epsilon_{t-k}$, where $\mu=E\left(Y_{t}\right)$, $\xi_{i}$ 's are recursively calculated as $\xi_{0}=1$, $\xi_{k}=\sum_{\ell=0}^{k-1} \xi_{\ell} \phi_{k-\ell}$ for $k=1,2, \ldots$ Moreover, $\xi_{k}$ 's are absolute summable.

For the long memory property of $Y_{t}$, we need the following condition:

(A2) For generic constants $c, \beta_{j} \sim c \lambda^{j}$ for some $|\lambda|<1$ and $h_{j} \sim c \omega^{j}$ for some $\omega>1$. Here, we write $a_{j} \sim b_{j}$ to denote $a_{j}=b_{j}+o\left(b_{j}\right)$.

According to Hwang and Shin (2014), the long-memory property of the HAR $(\infty)$ model has been investigated, which are stated in Propositions 2 and 3. In particular, Proposition 3 tells us that, given $h_{j} \sim c \omega^{j}, \mathrm{HAR}(\infty)$ model is of long-memory with algebraically decreasing ACF if and only if $\beta_{j}$ decreases exponentially.

Proposition 2. Under conditions (A1) and (A2), for $\rho=(\log \omega-\log |\lambda|) / \log \omega>1$ and generic constants $c$, we have $(i)\left|\xi_{k}\right| \sim c k^{-\rho}$ and (ii) $\gamma_{k}=\operatorname{Cov}\left(Y_{t}, Y_{t+k}\right)=\sigma_{\epsilon}^{2} \sum_{i=0}^{\infty} \xi_{i} \xi_{i+k} \sim c k^{-\rho}$.

Proposition 3. Assume (Al), $h_{j} \sim c \omega^{j}$ for some $\omega>1$, and $\left|\xi_{k}\right| \sim c k^{-\rho}$ for some $\rho>1$, then $\beta_{j} \sim c \lambda^{j}$ for $\lambda=\omega^{1-\rho} \in(0,1)$.

Estimation theories were provided by Hwang and Shin (2014) in which the infinite-order HAR model in (2.3) is estimated by a finite $p^{\text {th }}$ order model in (2.1) with $p$ increasing as sample size increases. They proved consistency and limiting normality of the OLSE of the HAR coefficients. 


\subsection{Functional central limit theorem}

Recently, Lee (2014) has established a FCLT for the HAR( $\infty)$ model by showing that $f\left(Y_{t}\right)$ is an $L_{2}$ NED on $\left\{\epsilon_{t}\right\}$ where $f(x)=|x|^{v}$ or $f(x)=\operatorname{sign}(x) \cdot|x|^{v},(v>0)$. A mean break test is developed from the CUSUM of $Y_{t}-\mu$ and a variance break test is developed from the CUSUM of $Y_{t}^{2}-E\left[Y_{t}^{2}\right]$, which correspond to $v=1$ and $v=2$, respectively. For notational simplicity, we denote $y_{t}=f\left(Y_{t}\right)$.

We say that $\left\{y_{t}\right\}$ is $L_{2}$-NED on $\left\{\epsilon_{t}\right\}$ if $\left\{y_{t}\right\}$ satisfies

$$
\left\|y_{t}-E\left[y_{t} \mid \mathcal{F}_{t-\ell}^{t+\ell}\right]\right\|_{2} \leq c_{t} d(\ell)
$$

where $\mathcal{F}_{t-\ell}^{t+\ell}=\sigma\left\{\epsilon_{t-\ell}, \ldots, \epsilon_{t}, \epsilon_{t+1}, \ldots, \epsilon_{t+\ell}\right\}$ is a $\sigma$-algebra generated by $\left\{\epsilon_{t-\ell}, \ldots, \epsilon_{t}, \epsilon_{t+1}, \ldots, \epsilon_{t+\ell}\right\}, c_{t}$ is a sequence of positive constants and $d(\ell) \rightarrow 0$ as $\ell \rightarrow \infty$. Denote $\|x\|_{p}$ by $\left(E|x|^{p}\right)^{1 / p}$ for $1 \leq p<\infty$ and by $E|x|^{p}$ for $0<p<1$. Let $\sigma_{y}^{2}=\operatorname{Var}\left(y_{1}\right)+2 \sum_{t=1}^{\infty} \operatorname{Cov}\left(y_{1}, y_{t+1}\right)$.

Proposition 4. (Lee, 2014) Assume that (A1), (A2) and one of (a) $0<v<1, v(\rho-1)>1$, or (b) $v \geq 1, \rho>3$ holds. If $\left\|\epsilon_{t}\right\|_{2 v}<\infty$ and $\sigma_{y}>0$ for each case, then

$$
S_{y n}(z):=\frac{1}{\sigma_{y} \sqrt{n}} \sum_{t=1}^{[n z]}\left(y_{t}-E\left[y_{t}\right]\right) \stackrel{d}{\longrightarrow} B(z),
$$

where $y_{t}=\left|Y_{t}\right|^{\nu}$ or $y_{t}=\operatorname{sign}\left(Y_{t}\right) \cdot\left|Y_{t}\right|^{\nu}$ and $B(z)$ is a standard Brownian motion for $0 \leq z \leq 1$.

The FCLT in Proposition 4 requires conditions on $v, \rho$ which require $\gamma_{k} \sim c k^{2 d-1}, d<0$. This condition does not permit fractional integration $I(d)$ with $d \in(0,1 / 2)$ because $I(d)$ process has $\gamma_{k} \sim$ $c k^{2 d-1}$. Our break tests below depend on the FCLT and are not valid for long memory process with $\gamma_{k} \sim c k^{2 d-1}, d \in(0,1 / 2)$. We observe that, for $I(d)$ process $y_{t}$, according to Baillie (1996), the weak limit of $S_{y n}(z)$ is a fractional Brownian motion $B_{d}(z)$ depending on the fractional integration order $d$. Consequently, the usual break tests tend to be over-sized if $d \in(0,1 / 2)$. This makes it very difficult to distinguish between long memory and break; in addition, the literature provides no satisfactory test. Therefore, validity of our test only under $d<0$ is not a real disadvantage.

\subsection{Existing break tests}

Let a data set $\left\{Y_{t}: t=1, \ldots, n\right\}$ be given. There are two strategies of constructing tests for detecting breaks in the mean or variance of $Y_{t}$ during the data $\operatorname{span}\{1, \ldots, n\}$ : one is based on the cumulative sum of observed data and the other is based on the cumulative sum of HAR-residuals. The long memory of the original sample is addressed by a consistent long-run variance estimator in the first strategy and by an HAR regression in the second strategy.

The first strategy was considered by Lee (2014). Applying the FCLT in Proposition 4, Lee (2014) considered the CUSUM test for mean break and the CUSUM test for the variance break as well as derived the limiting distributions of the CUSUM tests. The mean break test and variance break test are

$$
\begin{array}{ll}
Q_{n}^{M}=\frac{1}{\hat{\sigma}_{y} \sqrt{n}} \sup _{0 \leq z \leq 1}\left|\sum_{t=1}^{[n z]}\left(y_{t}-\bar{y}_{n}\right)\right|, \quad y_{t}=Y_{t}, \\
Q_{n}^{V}=\frac{1}{\hat{\sigma}_{y} \sqrt{n}} \sup _{0 \leq z \leq 1}\left|\sum_{t=1}^{[n z]}\left(y_{t}-\bar{y}_{n}\right)\right|, \quad y_{t}=Y_{t}^{2},
\end{array}
$$


respectively, where $\bar{y}_{n}=(1 / n) \sum_{t=1}^{n} y_{t}$ and

$$
\hat{\sigma}_{y}^{2}:=\frac{1}{n} \sum_{t=1}^{n}\left(y_{t}-\bar{y}_{n}\right)^{2}+\frac{2}{n} \sum_{t=1}^{\ell}\left(1-\frac{t}{\ell+1}\right) \sum_{i=1}^{n-t}\left(y_{i}-\bar{y}_{n}\right)\left(y_{i+t}-\bar{y}_{n}\right), \quad \ell<n
$$

is a consistent estimator of the long-run variance $\lim _{n \rightarrow \infty} n \operatorname{Var}\left(\bar{y}_{n}\right)$ with bandwidth $\ell$.

The second strategy was considered by Hwang and Shin $(2013,2015)$. The HAR $(p)$ model (2.1) is estimated by OLS regression. Let $\hat{\beta}_{0}, \ldots, \hat{\beta}_{p}$ be the OLSE. The break tests are based on OLS residuals

$$
\hat{a}_{t}=Y_{t}-\hat{\beta}_{0}-\hat{\beta}_{1} Y_{t, h_{1}}-\cdots-\hat{\beta}_{p} Y_{t, h_{p}}, \quad t=h_{p}+1, \ldots, n
$$

as given by

$$
\begin{aligned}
& P_{n}^{M}=\frac{1}{\hat{\sigma}_{a} \sqrt{n-h_{p}}} \sup _{0 \leq z \leq 1}\left|\sum_{t=h_{p}+1}^{[n z]} \hat{a}_{t}\right|, \\
& P_{n}^{V}=\frac{1}{\hat{\sigma}_{b} \sqrt{n-h_{p}}} \sup _{0 \leq z \leq 1}\left|\sum_{t=h_{p}+1}^{[n z]} \hat{b}_{t}\right|, \quad \hat{b}_{t}=\left(\hat{a}_{t}^{2}-\hat{\sigma}_{a}^{2}\right),
\end{aligned}
$$

where

$$
\hat{\sigma}_{a}^{2}:=\frac{1}{\left(n-h_{p}\right)} \sum_{t=h_{p}+1}^{n} \hat{a}_{t}^{2}, \quad \hat{\sigma}_{b}^{2}:=\frac{1}{\left(n-h_{p}\right)} \sum_{t=h_{p}+1}^{n} \hat{b}_{t}^{2} .
$$

The tests $P_{n}^{M}$ and $P_{n}^{V}$ detect breaks in mean and variance of $Y_{t}, t=1, \ldots, n$, respectively.

Large values of $Q_{n}^{M}, Q_{n}^{V}, P_{n}^{M}$, and $P_{n}^{V}$ reject the null hypotheses of parameter constancy. Critical values of the tests can be obtained from the large sample null distributions of the tests. Under the null hypothesis of no break, according to Proposition 4 and the results of Hwang and Shin (2013, 2015), with consistent $\hat{\sigma}_{y}$, the limiting null distributions of $Q_{n}^{M}, Q_{n}^{V}, P_{n}^{M}$, and $P_{n}^{V}$ are all standard Brownian bridges as given by

$$
Q_{n}^{M}, Q_{n}^{V}, P_{n}^{M}, P_{n}^{V} \stackrel{d}{\longrightarrow} \sup _{0 \leq z \leq 1}\left|B^{0}(z)\right| \quad \text { as } n \rightarrow \infty,
$$

whose distribution function is given by

$$
\operatorname{Pr}\left(\sup _{0 \leq z \leq 1}\left|B^{0}(z)\right| \leq x\right)=\sum_{k=-\infty}^{\infty}(-1)^{k} e^{-2 k^{2} x^{2}},
$$

where $B^{0}(z):=B(z)-z B(1)$ is a standard Brownian bridge and $B(z)$ is a standard Brownian motion.

\section{Stationary bootstrap tests}

We construct SB tests and prove their asymptotic validity. Assume that sample $\left\{Y_{t}: t=1,2, \ldots, n\right\}$ is given. We apply the SB of Politis and Romano (1994) to the sample $\left\{Y_{t}: t=1,2, \ldots, n\right\}$ to produce a SB sample $\left\{Y_{t}^{*}: t=1,2, \ldots, n\right\}$.

SB versions $\left(Q_{n}^{* M}, Q_{n}^{* V}, P_{n}^{* M}, P_{n}^{* V}\right)$ are constructed from the SB sample $\left\{Y_{t}^{*}: t=1,2, \ldots, n\right\}$ in the same way that $\left(Q_{n}^{M}, Q_{n}^{V}, P_{n}^{M}, P_{n}^{V}\right)$ are constructed from the original sample $\left\{Y_{t}: t=1,2, \ldots, n\right\}$. 
Consistencies of the null bootstrapping distributions of $\left(Q_{n}^{* M}, Q_{n}^{* V}, P_{n}^{* M}, P_{n}^{* V}\right)$ are proved. This enables us to use the quantiles of the bootstrapping distributions as critical values for the tests $\left(Q_{n}^{M}, Q_{n}^{V}\right.$, $\left.P_{n}^{M}, P_{n}^{V}\right)$. The tests with SB critical values will be called stationary bootstrap tests in the sequel. Consistencies of the SB tests are proved under alternatives of mean or variance changes.

We briefly describe how to construct SB sample from the original sample. Let $\left\{I_{1}, I_{2}, \ldots\right\}$ be independent uniform random variables on $\{1,2, \ldots, n\}$. Let $\left\{L_{1}: L_{2}, \ldots\right\}$ be independent geometric random variables with mean $1 / \varrho$, independent of $\left\{I_{1}, I_{2}, \ldots\right\}$. For observations $\left\{Y_{t}: t=1,2, \ldots, n\right\}$, consider periodic extensions $\left\{Y_{n, i}: i \geq 1\right\}$ by wrapping the sample in a circle with $i=n q+t$ for some $q$ and $1 \leq t \leq n$. Define the blocks $\mathcal{B}\left(I_{j}, L_{j}\right)$, starting $Y_{n, I_{j}}$ of block length $L_{j}$. Let $\kappa=\inf \{k \geq 1$ : $\left.L_{1}+\cdots+L_{k} \geq n\right\}$. Then combine the $\kappa$ blocks $\mathcal{B}\left(I_{1}, L_{1}\right), \ldots, \mathcal{B}\left(I_{k}, L_{\kappa}\right)$ and take the first $n$ elements to get the bootstrap sample $\left\{Y_{t}^{*}: t=1,2, \ldots, n\right\}$.

\subsection{Consistencies of the null distributions of $Q_{n}^{* M}$ and $Q_{n}^{* V}$}

The stationary bootstrap versions of $Q_{n}^{M}$ and $Q_{n}^{V}$ are

$$
\begin{array}{ll}
Q_{n}^{* M}=\frac{1}{\hat{\sigma}_{y}^{*} \sqrt{n}} \sup _{0 \leq z \leq 1}\left|\sum_{t=1}^{[n z]}\left(y_{t}^{*}-\bar{y}_{n}^{*}\right)\right|, \quad y_{t}^{*}=Y_{t}^{*}, \\
Q_{n}^{* V}=\frac{1}{\hat{\sigma}_{y}^{*} \sqrt{n}} \sup _{0 \leq z \leq 1}\left|\sum_{t=1}^{[n z]}\left(y_{t}^{*}-\bar{y}_{n}^{*}\right)\right|, \quad y_{t}^{*}=Y_{t}^{* 2},
\end{array}
$$

where

$$
\bar{y}_{n}^{*}=\frac{1}{n} \sum_{t=1}^{n} y_{t}^{*}, \quad \hat{\sigma}_{y}^{* 2}:=\frac{1}{n} \sum_{t=1}^{n}\left(y_{t}^{*}-\bar{y}_{n}^{*}\right)^{2}+\frac{2}{n} \sum_{t=1}^{\ell}\left(1-\frac{t}{\ell+1}\right) \sum_{i=1}^{n-t}\left(y_{i}^{*}-\bar{y}_{n}^{*}\right)\left(y_{i+t}^{*}-\bar{y}_{n}^{*}\right), \quad \ell<n .
$$

In order to prove consistencies of the null distributions of $Q_{n}^{* M}$ and $Q_{n}^{* V}$, we first establish a FCLT for the SB CUSUM

$$
S_{y n}^{*}(z):=\frac{1}{\sigma_{y, n}^{*} \sqrt{n}} \sum_{t=1}^{[n z]}\left(y_{t}^{*}-E^{*}\left(y_{t}^{*}\right)\right), \quad y_{t}^{*}=Y_{t}^{*} \text { or } Y_{t}^{* 2}
$$

for $0 \leq z \leq 1$, where

$$
\sigma_{y, n}^{* 2}:=\operatorname{Var}^{*}\left(\frac{1}{\sqrt{n}} \sum_{t=1}^{n} y_{t}^{*}\right)
$$

and $E^{*}$ and $\operatorname{Var}^{*}$ denote the expectation and variance conditional on sample $\left\{Y_{t}: t=1,2, \ldots, n\right\}$. In the following theorems, the bootstrap version $S_{y n}^{*}(z)$ of the cumulative sum $S_{y n}(z)$ is shown to converge to the standard Brownian motion as in Theorem 1 below, from which we obtain the consistencies of the null distributions of $Q_{n}^{* M}$ and $Q_{n}^{* V}$ as in Theorem 2 below.

Theorem 1. We assume the same conditions as in Proposition 4. Under the null hypothesis of no break, if $\varrho \rightarrow 0$ and $\sqrt{n} \varrho \rightarrow \infty$, then, as $n \rightarrow \infty$,

$$
S_{y n}^{*}(\cdot) \stackrel{d^{*}}{\longrightarrow} B(\cdot) \text { in probability, }
$$


where $\stackrel{d^{*}}{\longrightarrow}$ denotes convergence in distribution conditional on the sample $\left\{Y_{1}, \ldots, Y_{n}\right\}$.

Theorem 2. Assume the same conditions of Theorem 1. Assume further that the bandwidth $\ell$ is chosen so that $\hat{\sigma}_{y}$ is consistent. Then, as $n \rightarrow \infty$,

$$
Q_{n}^{* M}, Q_{n}^{* V} \stackrel{d^{*}}{\longrightarrow} \sup _{0 \leq z \leq 1}\left|B^{0}(z)\right| \quad \text { in probability. }
$$

\subsection{Consistencies of the null distributions of $P_{n}^{* M}$ and $P_{n}^{* V}$}

Let $\hat{\beta}_{0}^{*}, \ldots, \hat{\beta}_{p}^{*}$ be the OLSE constructed from the SB sample $\left\{Y_{1}^{*}, \ldots, Y_{n}^{*}\right\}$. Let

$$
\begin{aligned}
\hat{a}_{t}^{*} & =Y_{t}^{*}-\hat{\beta}_{0}^{*}-\hat{\beta}_{1}^{*} Y_{t, h_{1}}^{*}-\cdots-\hat{\beta}_{p}^{*} Y_{t, h_{p}}^{*}, \quad \hat{b}_{t}^{*}=\hat{a}_{t}^{* 2}-\hat{\sigma}_{a}^{* 2}, \quad t=h_{p}+1, \ldots, n, \\
\hat{\sigma}_{a}^{* 2} & :=\frac{1}{n-h_{p}} \sum_{t=h_{p}+1}^{n} \hat{a}_{t}^{* 2}, \quad \hat{\sigma}_{b}^{* 2}:=\frac{1}{n-h_{p}} \sum_{t=h_{p}+1}^{n} \hat{b}_{t}^{* 2}, \\
\sigma_{a n}^{* 2} & :=\operatorname{Var}^{*}\left(\frac{1}{\sqrt{n-h_{p}}} \sum_{t=h_{p}+1}^{n} \hat{a}_{t}^{*}\right), \quad \sigma_{b n}^{* 2}:=\operatorname{Var}^{*}\left(\frac{1}{\sqrt{n-h_{p}}} \sum_{t=h_{p}+1}^{n} \hat{b}_{t}^{*}\right) .
\end{aligned}
$$

The SB versions $P_{n}^{* M}$ and $P_{n}^{* V}$ of $P_{n}^{M}$ and $P_{n}^{V}$ are

$$
P_{n}^{* M}=\frac{1}{\hat{\sigma}_{a}^{*} \sqrt{n-h_{p}}} \sup _{0 \leq z \leq 1}\left|\sum_{t=h_{p}+1}^{[n z]} \hat{a}_{t}^{*}\right|, \quad P_{n}^{* V}=\frac{1}{\hat{\sigma}_{b}^{*} \sqrt{n-h_{p}}} \sup _{0 \leq z \leq 1}\left|\sum_{t=h_{p}+1}^{[n z]} \hat{b}_{t}^{*}\right| .
$$

Let

$$
S_{a n}^{*}(z):=\frac{1}{\sigma_{a n}^{*} \sqrt{n-h_{p}}} \sum_{t=h_{p}+1}^{[n z]}\left(\hat{a}_{t}^{*}-E^{*}\left(\hat{a}_{t}^{*}\right)\right), \quad S_{b n}^{*}(z):=\frac{1}{\sigma_{b n}^{*} \sqrt{n-h_{p}}} \sum_{t=h_{p}+1}^{[n z]}\left(\hat{b}_{t}^{*}-E^{*}\left(\hat{b}_{t}^{*}\right)\right)
$$

for $0 \leq z \leq 1$, where $E^{*}\left(\hat{a}_{t}^{*}\right)=E^{*}\left(\hat{b}_{t}^{*}\right)=0$. In the following theorems, the bootstrap versions $S_{a n}^{*}(z)$ and $S_{b n}^{*}(z)$ of the cumulative sums $S_{a n}(z)$ and $S_{b n}(z)$ are shown to converge to the standard Brownian motion as in Theorem 3 below, from which we get the consistencies of the null distributions of $P_{n}^{* M}$ and $P_{n}^{* V}$ as in Theorem 4 below.

Theorem 3. We assume the same conditions as in Proposition 4. Under the null hypothesis of no break, if $\varrho \rightarrow 0$, $\sqrt{n} \varrho \rightarrow \infty, p \rightarrow \infty$, and $p^{2+\epsilon}=O(n)$, then, as $n \rightarrow \infty$,

$$
S_{a n}^{*}(\cdot) \stackrel{d^{*}}{\longrightarrow} B^{0}(\cdot) \quad \text { in probability, } \quad S_{b n}^{*}(\cdot) \stackrel{d^{*}}{\longrightarrow} B^{0}(\cdot) \text { in probability. }
$$

Theorem 4. Assume the same conditions of Theorem 3. Then, as $n \rightarrow \infty$,

$$
P_{n}^{* M}, \quad P_{n}^{* V} \stackrel{d^{*}}{\longrightarrow} \sup _{0 \leq z \leq 1}\left|B^{0}(z)\right| \quad \text { in probability. }
$$




\subsection{Bootstrap tests for structural changes}

Thanks to the consistencies in Theorems 2 and 4, asymptotic critical values of mean break tests $Q_{n}^{M}$ and $P_{n}^{M}$ and variance break tests $Q_{n}^{V}$ and $P_{n}^{V}$ can be obtained from the distributions of the bootstrap statistics $Q_{n}^{* M}, P_{n}^{* M}, Q_{n}^{* V}$, and $P_{n}^{* V}$ instead of the large sample distribution in (2.5) and (2.6).

Let $R_{n}$ be one of $Q_{n}^{M}, P_{n}^{M}, Q_{n}^{V}$, and $P_{n}^{V}$ and let $R_{n}^{*}$ be the corresponding SB version. We can construct level- $\alpha$ bootstrap critical value $R_{n}^{*}(\alpha)$ as the $\alpha^{\text {th }}$ empirical quantile of the $m$ independent stationary bootstrap test values $\left\{R_{n}^{*}=R_{n}^{*(i)}, i=1, \ldots, m\right\}, \alpha \in(0,1)$. Now, SB test is: reject the null hypothesis of mean (or variance) constancy if $R_{n}$ is larger than $R_{n}^{*}(\alpha)$.

Consistencies of the stationary bootstrap tests will be proved under alternative hypotheses of a single mean break at time $t_{0} \in\{1, \ldots, n\}$

$$
E\left(Y_{t}\right)=\left\{\begin{array}{ll}
\mu_{(1)}, & t<t_{0}, \\
\mu_{(2)}, & t_{0} \leq t \leq n ;
\end{array} \quad \mu_{(1)} \neq \mu_{(2)} ; 0<\lim \inf _{n \rightarrow \infty} \frac{t_{0}}{n} \leq \lim \sup _{n \rightarrow \infty} \frac{t_{0}}{n}<1\right.
$$

or of a variance break at time $t_{0} \in\{1, \ldots, n\}$

$$
E\left(a_{t}^{2}\right)=\left\{\begin{array}{ll}
\sigma_{(1)}^{2}, & t<t_{0}, \\
\sigma_{(2)}^{2}, & t_{0} \leq t \leq n ;
\end{array} \quad \sigma_{(1)}^{2} \neq \sigma_{(2)}^{2} ; 0<\lim \inf _{n \rightarrow \infty} \frac{t_{0}}{n} \leq \lim \sup _{n \rightarrow \infty} \frac{t_{0}}{n}<1 .\right.
$$

In the followings, $\operatorname{Pr}^{*}$ denotes the conditional probability given on sample $\left\{Y_{t}: t=1,2, \ldots, n\right\}$.

Theorem 5. We assume the same conditions as in Theorem 2 except for the condition of no break. Let $\alpha \in(0,1)$. If (3.1) holds, then as $n \rightarrow \infty, \operatorname{Pr}^{*}\left[Q_{n}^{M}>Q_{n}^{* M}(\alpha)\right] \stackrel{p}{\longrightarrow} 1$. If (3.2) holds, then as $n \rightarrow \infty$, $\operatorname{Pr}^{*}\left[Q_{n}^{V}>Q_{n}^{* V}(\alpha)\right] \stackrel{p}{\longrightarrow} 1$.

Theorem 6. We assume the same conditions as in Theorem 4 except for the condition of no break. Let $\alpha \in(0,1)$. If (3.1) holds, then as $n \rightarrow \infty, \operatorname{Pr}^{*}\left[P_{n}^{M}>P_{n}^{* M}(\alpha)\right] \stackrel{p}{\longrightarrow}$ 1. If (3.2) holds, then as $n \rightarrow \infty$, $\operatorname{Pr}^{*}\left[P_{n}^{V}>P_{n}^{* V}(\alpha)\right] \stackrel{p}{\rightarrow} 1$.

\section{Monte-Carlo study}

A simulation experiment is conducted to investigate finite sample sizes and powers of the proposed tests for breaks in the memory parameters $\beta_{1}, \ldots, \beta_{p}$. Long-memory data are generated by approximating $\operatorname{HAR}(\infty)$ by $\operatorname{HAR}(7)$ model

$$
Y_{t}=\sum_{j=1}^{p} \beta_{j} Y_{t, h_{j}}+e_{t}, \quad p=7, t=1, \ldots, n,
$$

where $e_{t}$ is a sequence of independent standard normal errors. The sample size is set to $n=1,000$, $2,000,4,000$, which correspond roughly, 5 years, 10 years, and 20 years, respectively. The error term $e_{t}$ is set to independent standard normal variables. For power study of mean break tests, 0.125 is added to $Y_{t}$ for all $t>n / 2$. For power study of variance break tests, 1.08 is multiplied to $e_{t}$ for all $t>n / 2$. The parameters for the HAR model are chosen as in Table 1: $D_{1}$ and $D_{2}$ are HAR(7) with $\sum_{j=1}^{p} \beta_{j}=0.9$ with $\lambda=0.6,0.9$, respectively; $D_{3}$ and $D_{4}$ the historic HAR(3) models for RVs of the US S\&P500, and US T-bond, respectively, analyzed by Corsi (2009).

The normal errors $e_{t}$ are generated by RNNOA, a FORTRAN subroutine in IMSL. Data $Y_{t}$ are simulated from $t=-1,000$ with $Y_{t}=0, t<-10,00$. Data $Y_{1}, \ldots, Y_{n}$ are used for computing the 
Table 1: Parameters for data generating process (DGP)

\begin{tabular}{c|ccccccc|c}
\hline \hline DGP & $\beta_{1}$ & $\beta_{2}$ & $\beta_{3}$ & $\beta_{4}$ & $\beta_{5}$ & $\beta_{6}$ & $\beta_{7}$ & $h_{j}$ \\
\hline$D_{1}$ & 0.370 & 0.222 & 0.133 & 0.080 & 0.048 & 0.029 & 0.017 & $h_{j}=2^{j-1}, j=1, \ldots, 7$ \\
$D_{2}$ & 0.173 & 0.155 & 0.140 & 0.126 & 0.113 & 0.102 & 0.092 & $h_{j}=2^{j-1}, j=1, \ldots, 7$ \\
$D_{3}$ & 0.372 & 0.343 & 0.224 & & & & & $h_{1}=1, h_{2}=5, h_{3}=22$ \\
$D_{4}$ & 0.039 & 0.412 & 0.361 & & & & & $h_{1}=1, h_{2}=5, h_{3}=22$ \\
\hline \hline
\end{tabular}

Table 2: Rejection rates (\%) of the level 5\% mean break tests

\begin{tabular}{|c|c|c|c|c|c|c|c|c|c|c|c|c|c|c|c|c|c|c|}
\hline \multirow{3}{*}{ DGP } & \multirow{3}{*}{$n$} & \multirow{3}{*}{$\ell$} & \multicolumn{8}{|c|}{$D_{1}, \lambda=0.6$} & \multicolumn{8}{|c|}{$D_{2}, \lambda=0.9$} \\
\hline & & & \multicolumn{4}{|c|}{ Size } & \multicolumn{4}{|c|}{ Power } & \multicolumn{4}{|c|}{ Size } & \multicolumn{4}{|c|}{ Power } \\
\hline & & & $Q_{n}$ & $Q_{n}^{*}$ & $P_{n}$ & $P_{n}^{*}$ & $Q_{n}$ & $Q_{n}^{*}$ & $P_{n}$ & $P_{n}^{*}$ & $Q_{n}$ & $Q_{n}^{*}$ & $P_{n}$ & $P_{n}^{*}$ & $Q_{n}$ & $Q_{n}^{*}$ & $P_{n}$ & $P_{n}^{*}$ \\
\hline \multirow{12}{*}{$\operatorname{HAR}(7)$} & \multirow{4}{*}{1,000} & 0 & 92.5 & 4.6 & 3.2 & 3.9 & 99 & 29 & 39 & 25 & 95.7 & 4.7 & 3.1 & 3.7 & 100 & 29 & 39 & 25 \\
\hline & & 20 & 7.8 & 3.6 & - & - & 50 & 26 & - & - & 8.4 & 3.6 & - & - & 52 & 26 & - & - \\
\hline & & 80 & 2.4 & 4.5 & - & - & 26 & 28 & - & - & 2.4 & 4.4 & - & - & 26 & 28 & - & - \\
\hline & & 160 & 0.6 & 4.8 & - & - & 2 & 18 & - & - & 0.5 & 4.9 & - & - & 2 & 18 & - & - \\
\hline & \multirow{4}{*}{2,000} & 0 & 94.5 & 3.9 & 5.9 & 4.4 & 100 & 59 & 71 & 56 & 96.5 & 4.2 & 5.9 & 4.2 & 100 & 59 & 70 & 56 \\
\hline & & 20 & 8.7 & 3.5 & - & - & 76 & 49 & - & - & 10.0 & 3.2 & - & - & 77 & 49 & - & - \\
\hline & & 80 & 2.8 & 3.2 & - & - & 58 & 50 & - & - & 2.8 & 3.3 & - & - & 59 & 50 & - & - \\
\hline & & 160 & 2.8 & 5.4 & - & - & 39 & 45 & - & - & 2.7 & 5.5 & - & - & 39 & 45 & - & - \\
\hline & \multirow{4}{*}{4,000} & 0 & 94.7 & 4.2 & 5.1 & 4.2 & 100 & 89 & 95 & 89 & 98.1 & 4.4 & 5.1 & 4.2 & 100 & 89 & 95 & 89 \\
\hline & & 20 & 6.9 & 2.5 & - & - & 97 & 85 & - & - & 8.5 & 2.2 & - & - & 97 & 85 & - & - \\
\hline & & 80 & 3.6 & 3.9 & - & - & 93 & 87 & - & - & 3.6 & 3.9 & - & - & 94 & 87 & - & - \\
\hline & & 160 & 4.3 & 5.5 & - & - & 88 & 84 & - & - & 4.2 & 5.7 & - & - & 88 & 84 & - & - \\
\hline \multirow{3}{*}{ DGP } & \multirow{3}{*}{$n$} & \multirow{3}{*}{$\ell$} & \multicolumn{8}{|c|}{$D_{3}, \mathrm{~S} \& \mathrm{P} 500$} & \multicolumn{8}{|c|}{$D_{4}$, US T-Bond } \\
\hline & & & \multicolumn{4}{|c|}{ Size } & \multicolumn{4}{|c|}{ Power } & \multicolumn{4}{|c|}{ Size } & \multicolumn{4}{|c|}{ Power } \\
\hline & & & $Q_{n}$ & $Q_{n}^{*}$ & $P_{n}$ & $P_{n}^{*}$ & $Q_{n}$ & $Q_{n}^{*}$ & $P_{n}$ & $P_{n}^{*}$ & $Q_{n}$ & $Q_{n}^{*}$ & $P_{n}$ & $P_{n}^{*}$ & $Q_{n}$ & $Q_{n}^{*}$ & $P_{n}$ & $P_{n}^{*}$ \\
\hline \multirow{12}{*}{$\begin{array}{l}\text { Historic } \\
\operatorname{HAR}(3)\end{array}$} & \multirow{4}{*}{1,000} & 0 & 100.0 & 13.8 & 1.1 & 4.2 & 100 & 55 & 3 & 7 & 98.8 & 8.0 & 2.1 & 3.3 & 100 & 41 & 11 & 11 \\
\hline & & 20 & 67.7 & 9.7 & - & - & 93 & 50 & - & - & 44.2 & 6.3 & - & - & 85 & 40 & - & - \\
\hline & & 80 & 13.7 & 7.6 & - & - & 57 & 34 & - & - & 6.6 & 5.2 & - & - & 42 & 30 & - & - \\
\hline & & 160 & 0.1 & 3.4 & - & - & 0 & 11 & - & - & 0.1 & 4.0 & - & 0.0 & 1 & 14 & - & - \\
\hline & \multirow{5}{*}{2,000} & 0 & 100.0 & 13.0 & 3.6 & 5.2 & 100 & 80 & 17 & 26 & 99.7 & 7.3 & 4.9 & 5.7 & 100 & 68 & 49 & 47 \\
\hline & & 20 & 74.0 & 6.9 & - & - & 99 & 65 & - & - & 46.4 & 4.2 & - & - & 96 & 53 & - & - \\
\hline & & 80 & 17.4 & 3.6 & - & - & 84 & 51 & - & - & 7.0 & 2.6 & - & - & 73 & 46 & - & - \\
\hline & & 160 & 3.9 & 4.6 & - & - & 52 & 43 & - & - & 2.7 & 4.8 & - & - & 43 & 44 & - & - \\
\hline & & 0 & 100.0 & 10.6 & 3.3 & 5.5 & 100 & 96 & 74 & 80 & 100.0 & 6.9 & 4.5 & 5.1 & 100 & 93 & 89 & 87 \\
\hline & & 20 & 70.8 & 2.2 & - & - & 100 & 83 & - & - & 42.2 & 1.8 & - & - & 100 & 79 & - & - \\
\hline & 4,000 & 80 & 15.5 & 2.8 & - & - & 99 & 82 & - & - & 7.9 & 2.9 & - & - & 97 & 83 & - & - \\
\hline & & 160 & 7.4 & 3.8 & - & - & 93 & 82 & - & - & 5.6 & 4.1 & - & - & 91 & 83 & - & - \\
\hline
\end{tabular}

Note: Number of replications $=1,000$, number of bootstrap replication $=1,000$.

$\mathrm{DGP}=$ data generating process; HAR $=$ heterogeneous autoregressive.

mean break test statistics $Q_{n}^{M}, Q_{n}^{* M}, P_{n}^{M}, P_{n}^{* M}$ and the variance break test statistics $Q_{n}^{V}, Q_{n}^{* V}, P_{n}^{V}, P_{n}^{* V}$. For $Q_{n}^{M}, Q_{n}^{* M}, Q_{n}^{V}, Q_{n}^{* V}$ based on the CUSUM of $Y_{t}$ or $Y_{t}^{2}$, the bandwidth parameter for the longrun variance estimate (2.4) is chosen to be $\ell=i_{\ell}(n / 1000)^{1 / 4}, i_{\ell}=0,20,80,160$. We use the $1 / 4$-order bandwidth because it is generally recommended to be good for longrun variance estimate, see for example, Schwert (1989). A wide range of values of $\ell$ is chosen because these tests are very sensitive to $\ell$. For the $P$-statistics $P_{n}^{M}, P_{n}^{* M}, P_{n}^{V}, P_{n}^{* V}$ based on the residuals of HAR fitting, the residuals are computed from $\operatorname{HAR}(p)$ model, $p=2,3,4$.

For computing the stationary bootstrap tests $Q_{n}^{* M}, P_{n}^{* M} Q_{n}^{* V}, P_{n}^{* V}$, the block length parameter is chosen set to $\varrho=0.005(n / 1000)^{-1 / 3}$ so that the mean block length is $\varrho^{-1}$. The third order parameter is chosen because it is usually optimal for parameter estimators based on block bootstrapping, see for example Bühlmann (2002). 
Table 3: Rejection rates (\%) of the level 5\% variance break tests

\begin{tabular}{|c|c|c|c|c|c|c|c|c|c|c|c|c|c|c|c|c|c|c|}
\hline \multirow{3}{*}{ DGP } & \multirow{3}{*}{$n$} & \multirow{3}{*}{$\ell$} & \multicolumn{8}{|c|}{$D_{1}, \lambda=0.6$} & \multicolumn{8}{|c|}{$D_{2}, \lambda=0.9$} \\
\hline & & & \multicolumn{4}{|c|}{ Size } & \multicolumn{4}{|c|}{ Power } & \multicolumn{4}{|c|}{ Size } & \multicolumn{4}{|c|}{ Power } \\
\hline & & & $Q_{n}$ & $Q_{n}^{*}$ & $P_{n}$ & $P_{n}^{*}$ & $Q_{n}$ & $Q_{n}^{*}$ & $\overline{P_{n}}$ & $P_{n}^{*}$ & $Q_{n}$ & $Q_{n}^{*}$ & $P_{n}$ & $P_{n}^{*}$ & $Q_{n}$ & $Q_{n}^{*}$ & $\overline{P_{n}}$ & $P_{n}^{*}$ \\
\hline & & 0 & 54.0 & 3.9 & 4.1 & 3.4 & 65 & 9 & 32 & 19 & 66.0 & 3.7 & 4.0 & 3.8 & 73 & 7 & 32 & 19 \\
\hline & 1000 & 20 & 5.0 & 4.3 & - & - & 13 & 8 & - & - & 5.1 & 4.2 & - & - & 10 & 7 & - & - \\
\hline & 1,000 & 80 & 2.4 & 4.7 & - & - & 5 & 8 & - & - & 2.4 & 4.8 & - & - & 4 & 7 & - & - \\
\hline & & 160 & 0.4 & 5.7 & - & - & 1 & 8 & - & - & 0.2 & 5.8 & - & - & 1 & 8 & - & - \\
\hline cline $2-22$ & \multirow{4}{*}{2,000} & 0 & 55.6 & 3.1 & 4.9 & 3.1 & 79 & 16 & 61 & 45 & 66.9 & 2.9 & 4.9 & 3.1 & 83 & 13 & 61 & 45 \\
\hline \multirow{3}{*}{$\operatorname{HAR}(7)$} & & 20 & 4.0 & 2.3 & - & - & 21 & 11 & - & - & 4.1 & 2.6 & - & - & 18 & 9 & - & - \\
\hline & & 80 & 3.1 & 3.5 & - & - & 13 & 14 & - & - & 2.9 & 3.3 & - & - & 11 & 11 & - & - \\
\hline & & 160 & 1.7 & 4.4 & - & - & 9 & 15 & - & - & 1.7 & 4.0 & - & - & 7 & 13 & - & - \\
\hline \multirow[t]{4}{*}{ cline $2-22$} & \multirow{4}{*}{4,000} & 0 & 60.1 & 3.3 & 3.9 & 3.5 & 88 & 31 & 89 & 80 & 72.3 & 3.2 & 3.9 & 3.2 & 89 & 26 & 89 & 80 \\
\hline & & 20 & 4.8 & 2.9 & - & - & 39 & 27 & - & - & 5.2 & 2.9 & - & - & 33 & 22 & - & - \\
\hline & & 80 & 4.0 & 4.0 & - & - & 34 & 30 & - & - & 4.0 & 4.0 & - & - & 28 & 26 & - & - \\
\hline & & 160 & 2.9 & 4.3 & - & - & 28 & 29 & - & - & 2.6 & 4.1 & - & - & 25 & 26 & - & - \\
\hline \multirow{3}{*}{ DGP } & \multirow{3}{*}{$n$} & \multirow{3}{*}{$\ell$} & \multicolumn{8}{|c|}{$D_{3}, \mathrm{~S} \& \mathrm{P} 500$} & \multicolumn{8}{|c|}{$D_{4}$, US T-Bond } \\
\hline & & & \multicolumn{4}{|c|}{ Size } & \multicolumn{4}{|c|}{ Power } & \multicolumn{4}{|c|}{ Size } & \multicolumn{4}{|c|}{ Power } \\
\hline & & & $Q_{n}$ & $Q_{n}^{*}$ & $P_{n}$ & $P_{n}^{*}$ & $Q_{n}$ & $Q_{n}^{*}$ & $P_{n}$ & $P_{n}^{*}$ & $Q_{n}$ & $Q_{n}^{*}$ & $P_{n}$ & $P_{n}^{*}$ & $Q_{n}$ & $Q_{n}^{*}$ & $P_{n}$ & $P_{n}^{*}$ \\
\hline \multirow{12}{*}{$\begin{array}{l}\text { Historic } \\
\text { HAR(3) }\end{array}$} & \multirow{4}{*}{1,000} & 0 & 98.5 & 7.4 & 4.1 & 2.7 & 98 & 7 & 30 & 16 & 47.1 & 4.0 & 4.1 & 2.8 & 64 & 8 & 31 & 16 \\
\hline & & 20 & 38.7 & 6.5 & - & - & 38 & 8 & - & - & 14.2 & 4.5 & - & - & 25 & 10 & - & - \\
\hline & & 80 & 3.4 & 4.9 & - & - & 4 & 5 & - & - & 2.2 & 4.6 & - & - & 6 & 7 & - & - \\
\hline & & 160 & 0.1 & 4.0 & - & - & 0 & 4 & - & - & 0.7 & 3.7 & - & - & 1 & 7 & - & - \\
\hline & \multirow{4}{*}{2,000} & 0 & 99.6 & 6.0 & 3.9 & 2.3 & 100 & 7 & 60 & 41 & 60.1 & 3.9 & 4.2 & 2.8 & 77 & 15 & 60 & 42 \\
\hline & & 20 & 35.8 & 2.3 & - & - & 46 & 5 & - & - & 11.8 & 1.2 & - & - & 37 & 12 & - & - \\
\hline & & 80 & 6.4 & 3.9 & - & - & 7 & 4 & - & - & 3.5 & 3.6 & - & - & 16 & 12 & - & - \\
\hline & & 160 & 1.2 & 2.7 & - & - & 2 & 5 & - & - & 1.8 & 4.0 & - & - & 8 & 13 & - & - \\
\hline & & 0 & 99.9 & 5.5 & 3.6 & 2.3 & 100 & 9 & 88 & 78 & 66.0 & 3.2 & 3.7 & 2.8 & 87 & 29 & 88 & 79 \\
\hline & & 20 & 40.9 & 2.1 & - & - & 46 & 3 & - & - & 14.9 & 2.2 & - & - & 55 & 20 & - & - \\
\hline & 4,000 & 80 & 7.3 & 2.4 & - & - & 11 & 5 & - & - & 5.3 & 3.7 & - & - & 33 & 26 & - & - \\
\hline & & 160 & 2.0 & 3.2 & - & - & 5 & 5 & - & - & 2.1 & 3.1 & - & - & 25 & 25 & - & - \\
\hline
\end{tabular}

Note: Number of replications $=1,000$, number of bootstrap replication $=1,000$

$\mathrm{DGP}=$ data generating process; HAR = heterogeneous autoregressive.

Tables 2 and 3 report sizes and powers of the mean break tests and volatility break tests, respectively, which are based on 1,000 independent replications with $m=1,000$ bootstrapping samples. In computing the $P$-tests and $P^{*}$-tests, we first consider HAR(3) fitting because HAR(3) model is usually used in practice for analyzing realized volatilities. We observe the following.

1. SB improves sizes of $Q_{n}^{M}, Q_{n}^{V}, P_{n}^{M}$ but does not improve size of $P_{n}^{V}$.

2. Among the four mean break tests $Q_{n}^{M}, Q_{n}^{* M}, P_{n}^{M}, P_{n}^{* M}$, the SB test $P_{n}^{* M}$ based on HAR residual is the best.

3. Among the four variance break tests $Q_{n}^{V}, Q_{n}^{* V}, P_{n}^{V}, P_{n}^{* V}$, the normal test $P_{n}^{V}$ based on HAR residual is the best.

We next investigate the performance of the $P$-tests and $P^{*}$-tests in terms of the HAR estimation order $p$. Tables 4 and 5 report sizes and powers of the tests which are constructed under the same setup used for Tables 2 and 3. We observe the following.

1. For mean break test, we see, for $p=2$ of (relatively) under-specified order, the $P$-tests tend to be oversized, especially for $D_{3}$ and $D_{4}$ of the historical DGPs; the SB mitigate over-size for $D_{1}$ and $D_{2}$ but not for $D_{3}$ and $D_{4}$. 
Table 4: Rejection rates (\%) of the level 5\% mean break tests $P_{n}^{M}$ and $P_{n}^{* M}$ based on $\operatorname{HAR}(p)$ fittings

\begin{tabular}{|c|c|c|c|c|c|c|c|c|c|c|c|c|c|c|c|c|c|}
\hline \multirow{3}{*}{$p$} & \multirow{3}{*}{$n$} & \multicolumn{4}{|c|}{$D_{1}, \lambda=0.6$} & \multicolumn{4}{|c|}{$D_{2}, \lambda=0.9$} & \multicolumn{4}{|c|}{$D_{3}, \mathrm{~S} \& \mathrm{P} 500$} & \multicolumn{4}{|c|}{$D_{4}$, US T-Bond } \\
\hline & & \multicolumn{2}{|c|}{ Size } & \multicolumn{2}{|c|}{ Power } & \multicolumn{2}{|c|}{ Size } & \multicolumn{2}{|c|}{ Power } & \multicolumn{2}{|c|}{ Size } & \multicolumn{2}{|c|}{ Power } & \multicolumn{2}{|c|}{ Size } & \multicolumn{2}{|c|}{ Power } \\
\hline & & $P_{n}$ & $P_{n}^{*}$ & $P_{n}$ & $P_{n}^{*}$ & $P_{n}$ & $P_{n}^{*}$ & $P_{n}$ & $P_{n}^{*}$ & $P_{n}$ & $P_{n}^{*}$ & $P_{n}$ & $P_{n}^{*}$ & $P_{n}$ & $P_{n}^{*}$ & $P_{n}$ & $P_{n}^{*}$ \\
\hline \multirow{3}{*}{2} & 1,000 & 6.0 & 3.0 & 46 & 38 & 6.8 & 3.0 & 48 & 37 & 30.7 & 20.3 & 71 & 66 & 34.3 & 12.9 & 80 & 61 \\
\hline & 2,000 & 8.2 & 5.1 & 76 & 67 & 8.1 & 5.1 & 77 & 66 & 39.5 & 21.2 & 94 & 87 & 43.6 & 13.8 & 95 & 82 \\
\hline & 4,000 & 7.4 & 4.1 & 96 & 95 & 7.6 & 3.9 & 96 & 94 & 39.5 & 18.4 & 100 & 99 & 43.7 & 11.7 & 100 & 97 \\
\hline \multirow{3}{*}{3} & 1,000 & 3.2 & 3.9 & 39 & 25 & 3.1 & 3.7 & 39 & 25 & 1.1 & 4.2 & 3 & 7 & 2.1 & 3.3 & 11 & 11 \\
\hline & 2,000 & 5.9 & 4.4 & 71 & 56 & 5.9 & 4.2 & 70 & 56 & 3.6 & 5.2 & 17 & 26 & 4.9 & 5.7 & 49 & 47 \\
\hline & 4,000 & 5.1 & 4.2 & 95 & 89 & 5.1 & 4.2 & 95 & 89 & 3.3 & 5.5 & 74 & 80 & 4.5 & 5.1 & 89 & 87 \\
\hline \multirow{3}{*}{4} & 1,000 & 3.3 & 3.3 & 35 & 36 & 3.5 & 3.0 & 36 & 37 & 1.7 & 2.1 & 2 & 2 & 1.7 & 3.0 & 4 & 5 \\
\hline & 2,000 & 5.1 & 5.2 & 68 & 65 & 5.4 & 4.8 & 70 & 65 & 2.6 & 3.1 & 8 & 12 & 2.8 & 3.9 & 26 & 36 \\
\hline & 4,000 & 4.4 & 3.7 & 95 & 94 & 4.5 & 3.9 & 95 & 94 & 2.7 & 4.0 & 57 & 72 & 3.4 & 4.3 & 83 & 88 \\
\hline
\end{tabular}

Note: Number of replications $=1000$, number of bootstrap replication $=1000$.

$\mathrm{HAR}=$ heterogeneous autoregressive.

Table 5: Rejection rates (\%) of the level 5\% variance break tests $P_{n}^{V}$ and $P_{n}^{* V}$ based on $\operatorname{HAR}(p)$ fittings

\begin{tabular}{|c|c|c|c|c|c|c|c|c|c|c|c|c|c|c|c|c|c|}
\hline \multirow{3}{*}{$p$} & \multirow{3}{*}{$n$} & \multicolumn{4}{|c|}{$D_{1}, \lambda=0.6$} & \multicolumn{4}{|c|}{$D_{2}, \lambda=0.9$} & \multicolumn{4}{|c|}{$D_{3}, \mathrm{~S} \& \mathrm{P} 500$} & \multicolumn{4}{|c|}{$D_{4}$, US T-Bond } \\
\hline & & \multicolumn{2}{|c|}{ Size } & \multicolumn{2}{|c|}{ Power } & \multicolumn{2}{|c|}{ Size } & \multicolumn{2}{|c|}{ Power } & \multicolumn{2}{|c|}{ Size } & \multicolumn{2}{|c|}{ Power } & \multicolumn{2}{|c|}{ Size } & \multicolumn{2}{|c|}{ Power } \\
\hline & & $P_{n}$ & $P_{n}^{*}$ & $P_{n}$ & $P_{n}^{*}$ & $P_{n}$ & $P_{n}^{*}$ & $P_{n}$ & $P_{n}^{*}$ & $P_{n}$ & $P_{n}^{*}$ & $P_{n}$ & $P_{n}^{*}$ & $P_{n}$ & $P_{n}^{*}$ & $\overline{P_{n}}$ & $P_{n}^{*}$ \\
\hline \multirow{3}{*}{2} & 1,000 & 5.3 & 3.6 & 31 & 25 & 5.2 & 3.7 & 31 & 26 & 5.3 & 3.1 & 31 & 23 & 5.5 & 3.3 & 31 & 25 \\
\hline & 2,000 & 4.5 & 4.0 & 59 & 53 & 4.7 & 3.7 & 59 & 53 & 4.8 & 3.5 & 58 & 49 & 5.2 & 3.9 & 59 & 50 \\
\hline & 4,000 & 5.3 & 4.6 & 89 & 88 & 5.3 & 4.5 & 89 & 88 & 5.3 & 3.7 & 88 & 84 & 5.3 & 4.4 & 88 & 86 \\
\hline \multirow{3}{*}{3} & 1,000 & 4.1 & 3.4 & 32 & 19 & 4.0 & 3.8 & 32 & 19 & 4.1 & 2.7 & 30 & 16 & 4.1 & 2.8 & 31 & 16 \\
\hline & 2,000 & 4.9 & 3.1 & 61 & 45 & 4.9 & 3.1 & 61 & 45 & 3.9 & 2.3 & 60 & 41 & 4.2 & 2.8 & 60 & 42 \\
\hline & 4,000 & 3.9 & 3.5 & 89 & 80 & 3.9 & 3.2 & 89 & 80 & 3.6 & 2.3 & 88 & 78 & 3.7 & 2.8 & 88 & 79 \\
\hline \multirow{3}{*}{4} & 1,000 & 4.9 & 3.7 & 31 & 26 & 5.2 & 3.7 & 31 & 26 & 5.5 & 3.1 & 28 & 21 & 5.3 & 3.4 & 29 & 22 \\
\hline & 2,000 & 4.6 & 3.9 & 59 & 53 & 4.7 & 4.0 & 59 & 53 & 4.6 & 2.5 & 56 & 47 & 4.6 & 3.6 & 57 & 49 \\
\hline & 4,000 & 5.1 & 4.9 & 89 & 88 & 5.3 & 4.8 & 89 & 88 & 5.0 & 3.5 & 88 & 85 & 5.2 & 4.5 & 88 & 87 \\
\hline
\end{tabular}

Note: Number of replications $=1000$, number of bootstrap replication $=1000$.

$\mathrm{HAR}=$ heterogeneous autoregressive.

2. For the mean break test, we see the power of the test with $p=4$ of (relatively) over-specified order tend to be smaller than that with $p=3$.

3. For break test, there is no significance difference in size and power performances of tests with $p=2,3,4$.

From this experiment, we can say that SB considerably improve the sizes of the existing tests, especially mean break tests, without power loss. No substantial improvement is achieved for the variance break test $P_{n}^{V}$ by the SB because the size and power of variance break test $P_{n}^{V}$ is already robust against the bandwidth parameter $\ell$ or HAR estimation order $p$. The widely used HAR(3) fitting produces $P$-tests and $P^{*}$-tests with not worse performance than HAR(2) or HAR(4) fittings.

\section{Conclusion}

We established the stationary bootstrap functional central limit theorem (FCLT) for the HAR( $\infty)$ model, which is a genuine long-memory model for realized volatility in financial economics. The bootstrap version of the cumulative sum of the $\operatorname{HAR}(\infty)$ process is shown to converge to the standard Brownian motion. Applying the FCLT, under the null hypothesis of no break, we have established consistencies of the bootstrap null distributions of the SB CUSUM tests for structural mean change and for structural variance change. Consistencies of the stationary bootstrap tests are also established 
under alternative hypotheses of mean break and of variance break. Monte-Carlo simulation shows that SB improves size performance of existing tests especially for mean break tests.

\section{Appendix: Proofs}

Proof of Theorem 1: Proof is given in a similar way to that of Theorem 1 of Parker et al. (2006). Note that $E^{*} y_{t}^{*}=(1 / n) \sum_{t=1}^{n} y_{t}=\bar{y}_{n}$. For $t=1,2, \ldots, n$, let $K_{t}=\inf \left\{k: L_{1}+\cdots+L_{k} \geq t\right\}$ and note that $K_{n}=\kappa$. We observe

$$
\frac{1}{\sqrt{n}} \sum_{t=1}^{[n z]}\left(y_{t}^{*}-E^{*} y_{t}^{*}\right)=\frac{1}{\sqrt{n}}\left(\sum_{j=1}^{K_{[n z]}} \sum_{t=1}^{L_{j}}\left(y_{n, I_{j}+t-1}-\bar{y}_{n}\right)-\sum_{t=1}^{M_{[n z]}-[n z]}\left(y_{n, I_{[n z]}^{\prime}+L_{[n z]}^{\prime}-t}-\bar{y}_{n}\right)\right)
$$

where $M_{[n z]}=L_{1}+\cdots+L_{K_{[n z]}}, I_{[n z]}^{\prime}=I_{K_{[n z]}}$ and $L_{[n z]}^{\prime}=L_{K_{[n z]}}$. It is well-known, (see Politis and Romano (1994) or Hwang and Shin (2012)), that the last $n-\left(L_{1}+\cdots+L_{\kappa-1}\right)$ observations in the last block $\mathcal{B}\left(I_{\kappa}, L_{\kappa}\right)$ of the SB procedure does not affect the limiting distribution of the SB sample mean by the memoryless property of the geometric distribution, and thus in the same way we have $(1 / \sqrt{n}) \sum_{t=1}^{M_{[n z]}-[n z]}\left(y_{n, I_{[n z]}^{\prime}+L_{[n z]}^{\prime}-t}-\bar{y}_{n}\right) \stackrel{p}{\longrightarrow} 0$.

For $j=1,2, \ldots, \kappa$, let $\hat{U}_{j}=\sum_{t=1}^{L_{j}}\left(y_{n, I_{j}+t-1}-\bar{y}_{n}\right)$, i.e., the sum of centered $y_{n, t}$ s belonging to block $\mathcal{B}\left(I_{j}, L_{j}\right)$, and let $\hat{R}_{n}(z)=(1 / \sqrt{n}) \sum_{j=1}^{K_{[n z]}} \hat{U}_{j}$ for $1 \leq z \leq 1$. For the desired result, it suffices to show $\hat{R}_{n}(\cdot) \stackrel{d^{*}}{\longrightarrow} \sigma_{y} B(\cdot)$ in probability. Its proof is based on Theorem 13.5 of Billingsley (1999), which requires the convergence of the finite-dimensional distribution and the condition for the tightness of the partial sum process in the followings: for $0 \leq z_{1}<\cdots<z_{k} \leq 1$ in probability

$$
\left(\hat{R}_{n}\left(z_{1}\right), \ldots, \hat{R}_{n}\left(z_{k}\right)\right) \stackrel{d^{*}}{\longrightarrow} N(0, \Sigma),
$$

where $\Sigma=\left(\left(c_{i, j}\right)\right)_{i, j=1, \ldots, k}$ with $c_{i, j}=\sigma_{y}^{2} \min \left\{z_{i}, z_{j}\right\}$ and for $0 \leq z<u<r \leq 1$

$$
E^{*}\left(\left|\hat{R}_{n}(r)-\hat{R}_{n}(u)\right|^{2}\left|\hat{R}_{n}(u)-\hat{R}_{n}(z)\right|^{2}\right) \leq C(r-z)^{2} .
$$

Verifications of (A.1) and (A.2) can be given in the same way as those on pp. 627-628, (proofs of Eqs. (34) and (35)), of Parker et al. (2006). Note that Gonçalves and de Jong (2003) proved the first order asymptotic validity of the SB of the NED process for $\varrho \rightarrow 0$ and $\sqrt{n} \varrho \rightarrow \infty$, which can be applied to the process $y_{t}$ according to Lemmas 1 and 2 of Lee (2014), and thus the convergence of the SB variance of the NED process $y_{t}$ holds in probability as follows: $\sigma_{y, n}^{* 2}:=\operatorname{Var}^{*}\left((1 / \sqrt{n}) \sum_{t=1}^{n} y_{t}^{*}\right) \stackrel{p}{\longrightarrow} \sigma_{y}^{2}$. Hence we finish the proof of Theorem 1.

Proof of Theorem 2: It is obvious from the result in Theorem 1.

Proof of Theorem 3: Let $\tilde{a}_{t}=Y_{t}^{*}-\hat{\beta}_{0}-\sum_{j=1}^{p} \hat{\beta}_{j} Y_{t, h_{j}}^{*}$ and we mimic the model (2.1) by the stationary bootstrap sample $\left\{Y_{t}^{*}\right\}$ as follows:

$$
Y_{t}^{*}=\hat{\beta}_{0}+\sum_{j=1}^{p} \hat{\beta}_{j} Y_{t, h_{j}}^{*}+\tilde{a}_{t}
$$


Following the same arguments as in Step 1 of the proof of Theorem 2.4 by Hwang and Shin (2013), together with the asymptotic normality of the stationary sequence $\left\{\tilde{a}_{t}: t=1,2, \ldots\right\}$, we can show that

$$
\frac{1}{\sqrt{n-h_{p}}}\left(\sum_{t=h_{p}+1}^{[n z]} \tilde{a}_{t}-z \sum_{t=h_{p}+1}^{n} \tilde{a}_{t}\right) \stackrel{d^{*}}{\longrightarrow} \tilde{\sigma}_{a} B^{0}(z)
$$

where $\tilde{\sigma}_{a}^{2}=\operatorname{plim}_{n \rightarrow \infty} \operatorname{Var}^{*}\left(\sum_{t=1}^{n} \tilde{a}_{t} / \sqrt{n}\right)$.

The model (A.3) can be written as

$$
Y_{t}^{*}=\hat{\zeta}_{0}+\sum_{j=1}^{p} \hat{\beta}_{j}\left(Y_{t, h_{j}}^{*}-\hat{\mu}\right)+\tilde{a}_{t}
$$

where $\hat{\zeta}_{0}=\hat{\beta}_{0}+\sum_{j=1}^{p} \hat{\beta}_{j} \hat{\mu}$ and $\hat{\mu}=(1 / n) \sum_{t=1}^{n} Y_{t}$. By the same arguments as in Step 2 of the proof of Theorem 2.4 by Hwang and Shin (2013), we can obtain that

$$
\frac{1}{\sqrt{n-h_{p}}} \sum_{t=h_{p}+1}^{[n z]}\left(\hat{\beta}^{*}-\hat{\beta}\right)^{\prime} X_{t}^{*}=\frac{z}{\sqrt{n-h_{p}}} \sum_{t=h_{p}+1}^{n} \tilde{a}_{t}+o_{p}(1)
$$

where $X_{t}^{*}=\left(1, Y_{t, h_{1}}^{*}-\hat{\mu}, \ldots, Y_{t, h_{p}}^{*}-\hat{\mu}\right)^{\prime}, \hat{\beta}=\left(\hat{\zeta}_{0}, \hat{\beta}_{1}, \ldots, \hat{\beta}_{p}\right)^{\prime}$, and $\hat{\beta}^{*}=\left(\hat{\zeta}_{0}^{*}, \hat{\beta}_{1}^{*}, \ldots, \hat{\beta}_{p}^{*}\right)^{\prime}$ with $\hat{\zeta}_{0}^{*}=$ $\hat{\beta}_{0}^{*}+\sum_{j=1}^{p} \hat{\beta}_{j}^{*} \hat{\mu}$. The result in (A.5) is obtained by observing that

$$
\sqrt{n-h_{p}}\left(\hat{\beta}^{*}-\hat{\beta}\right)=\frac{1}{\sqrt{n-h_{p}}}\left[\begin{array}{ll}
1 & 0 \\
0 & \mathbf{R}
\end{array}\right]^{-1}\left[\begin{array}{c}
\sum_{t=h_{p}+1}^{n} \tilde{a}_{t} \\
\sum_{t=h_{p}+1}^{n} X_{t, 0}^{*} \tilde{a}_{t}
\end{array}\right]+o_{p}(1),
$$

where $\mathbf{R}$ is the $p \times p$ matrix with $(i, j)$-component $E^{*}\left[\left(Y_{t, h_{i}}^{*}-\hat{\mu}\right)\left(Y_{t, h_{j}}^{*}-\hat{\mu}\right)\right]$, and $X_{t, 0}^{*}=\left(Y_{t, h_{1}}^{*}-\hat{\mu}, \ldots, Y_{t, h_{p}}^{*}-\right.$ $\hat{\mu})^{\prime}$, and by the following convergence:

$$
\frac{1}{n-h_{p}} \sum_{t=h_{p}+1}^{[n z]} X_{t}^{*}=\frac{1}{n-h_{p}}\left([n z]-h_{p}, \sum_{t=h_{p}+1}^{[n z]} Y_{t, h_{i}}^{*}-\hat{\mu}, \ldots, \sum_{t=h_{p}+1}^{[n z]} Y_{t, h_{p}}^{*}-\hat{\mu}\right)^{\prime} \stackrel{p^{*}}{\rightarrow}(z, 0, \ldots, 0)^{\prime} .
$$

To prove the first convergence in distribution for $S_{a n}^{*}(\cdot)$ in Theorem 3, we note that

$$
\hat{a}_{t}^{*}=Y_{t}^{*}-\hat{\beta}^{* \prime} X_{t}^{*}, \quad \tilde{a}_{t}=Y_{t}^{*}-\hat{\beta}^{\prime} X_{t}^{*}
$$

and we observe, by (A.4) and (A.5)

$$
\begin{aligned}
\frac{1}{\sqrt{n-h_{p}}} \sum_{t=h_{p}+1}^{[n z]} \hat{a}_{t}^{*} & =\left(\frac{1}{\sqrt{n-h_{p}}} \sum_{t=h_{p}+1}^{[n z]} Y_{t}^{*}-\frac{1}{\sqrt{n-h_{p}}} \sum_{t=h_{p}+1}^{[n z]} \hat{\beta}^{\prime} X_{t}^{*}\right)-\frac{1}{\sqrt{n-h_{p}}} \sum_{t=h_{p}+1}^{[n z]}\left(\hat{\beta}^{*}-\hat{\beta}\right)^{\prime} X_{t}^{*} \\
& =\frac{1}{\sqrt{n-h_{p}}} \sum_{t=h_{p}+1}^{[n z]} \tilde{a}_{t}-\frac{z}{\sqrt{n-h_{p}}} \sum_{t=1}^{n} \tilde{a}_{t}+o_{p}(1) \stackrel{d^{*}}{\longrightarrow} \tilde{\sigma}_{a} B^{0}(z) .
\end{aligned}
$$

It is clear that $\sigma_{a n}^{* 2} \stackrel{p}{\longrightarrow} \tilde{\sigma}_{a}^{2}$ as $n \rightarrow \infty$. Thus we obtain the first desired convergence in distribution. 
To prove the second convergence in distribution for $S_{b n}^{*}(\cdot)$, in the model (A.3), we can show that, uniformly in $z$,

$$
\frac{1}{\sqrt{n-h_{p}}}\left(\sum_{t=h_{p}+1}^{[n z]} \hat{a}_{t}^{* 2}-\sum_{t=h_{p}+1}^{[n z]} \tilde{a}_{t}^{2}\right) \stackrel{p^{*}}{\longrightarrow} 0
$$

and letting $\xi_{t}=\tilde{a}_{t}^{2} / \tilde{\sigma}_{a}^{2}-1$,

$$
\frac{1}{\sqrt{n-h_{p}}}\left(\sum_{t=h_{p}+1}^{[n z]} \xi_{t}-z \sum_{t=h_{p}+1}^{n} \xi_{t}\right) \stackrel{d^{*}}{\longrightarrow} \frac{\phi^{\frac{1}{2}}}{\tilde{\sigma}_{a}^{2}} B^{0}(z),
$$

where $\phi=\phi^{1 / 2} \phi^{1 / 2}:=\tilde{\sigma}_{a}^{4} \lim _{n \rightarrow \infty} \operatorname{Var}\left(\sum_{t=1}^{n} \xi_{t} / \sqrt{n}\right)$. We can now observe that

$$
\begin{aligned}
\frac{1}{\sqrt{n-h_{p}}} \sum_{t=h_{p}+1}^{[n z]} \hat{b}_{t}^{*} & =\frac{1}{\sqrt{n-h_{p}}} \sum_{t=h_{p}+1}^{[n z]}\left(\hat{a}_{t}^{* 2}-\hat{\sigma}_{a}^{* 2}\right) \\
& =\frac{1}{\sqrt{n-h_{p}}}\left[\sum_{t=h_{p}+1}^{[n z]} \hat{a}_{t}^{* 2}-\frac{[n z]-h_{p}}{n-h_{p}} \sum_{t=h_{p}+1}^{n} \hat{a}_{t}^{* 2}\right] \\
& =\frac{1}{\sqrt{n-h_{p}}}\left[\sum_{t=h_{p}+1}^{[n z]} \tilde{a}_{t}^{2}-z \sum_{t=h_{p}+1}^{n} \tilde{a}_{t}^{2}\right]+o_{p}(1) \\
& =\frac{\tilde{\sigma}_{a}^{2}}{\sqrt{n-h_{p}}}\left[\sum_{t=h_{p}+1}^{[n z]}\left(\frac{\tilde{a}_{t}^{2}}{\tilde{\sigma}_{a}^{2}}-1\right)-z \sum_{t=h_{p}+1}^{n}\left(\frac{\tilde{a}_{t}^{2}}{\tilde{\sigma}_{a}^{2}}-1\right)\right]+o_{p}(1) \stackrel{d^{*}}{\longrightarrow} \phi^{\frac{1}{2}} B^{0}(z),
\end{aligned}
$$

and $\sigma_{b n}^{* 2} \stackrel{p}{\longrightarrow} \phi$ as $n \rightarrow \infty$. More detailed verifications can be given by the same arguments as in proof of Theorem 2.4 of Hwang and Shin (2015) along with their Lemmas 6.1 and 6.2, as applied to the model (A.3) above.

Proof of Theorem 4: It is obvious from the results in Theorem 3.

Proof of Theorems 5-6: Let $R_{n}$ be one of $Q_{n}^{M}, P_{n}^{M}, Q_{n}^{V}, P_{n}^{V}$ and let $R_{n}^{*}$ be the corresponding SB version. It suffices to show

$$
R_{n}^{*}=O_{p}(1) \quad \text { and } \quad R_{n} \rightarrow \quad \text { as } n \rightarrow \infty .
$$

First, for $R_{n}=Q_{n}^{M}$ with $y_{t}=Y_{t}$, to show the boundedness, we note that under the alternative, $y_{t}^{*}$ do not have structural changes because of the random block selection. We follow the same arguments as in the proof of Theorem 1. The block sums $\hat{U}_{j}$ in the proof of Theorem 1 are also iid under the alternative; therefore, the same arguments as those in proof of Theorem 1 provide a weak convergence of $Q_{n}^{* M}$ under the alternative and $Q_{n}^{* M}=O_{p}(1)$.

We show the second limiting in (A.6). Let $\sigma^{2}=\operatorname{Var}\left[y_{t}\right]$. Under the alternative hypothesis with break point $t_{0}$, we write $y_{t}=\mu_{(1)}+\sigma\left(\delta_{0} \mathbb{I}\left\{t>t_{0}\right\}+u_{t}\right)$ where $u_{t}$ is a sequence with mean zero and variance one, and $\delta_{0}=\left(\mu_{(2)}-\mu_{(1)}\right) / \sigma>0$. Let $Z_{n}(z)=\sum_{t=1}^{[n z]}\left(y_{t}-\bar{y}_{n}\right)$. We observe $Z_{n}(z)$ for $z \in\left[0, t_{0} / n\right]$ and $z \in\left(t_{0} / n, 1\right]$, respectively. It can be shown straightforward that

$$
Z_{n}(z)=\tilde{\sigma}\left(\sum_{t=1}^{[n z]} u_{t}-\frac{[n z]}{n} \sum_{t=1}^{n} u_{t}\right)+\tilde{\sigma} \lambda_{n}(z),
$$


where

$$
\lambda_{n}(z)= \begin{cases}-\delta_{0} \frac{[n z]\left(n-t_{0}\right)}{n}, & \text { if } 0 \leq[n z] \leq t_{0}, \\ -\delta_{0} \frac{t_{0}(n-[n z])}{n}, & \text { if } t_{0}<[n z] \leq n\end{cases}
$$

Noting that $Q_{n}^{M}=\sup _{0 \leq z \leq 1}\left|Z_{n}(z)\right| /\left(\hat{\sigma}_{y, n} \sqrt{n}\right)$, for the second desired limiting, we may show that $\sup _{0 \leq z \leq 1}\left|\lambda_{n}(z)\right| / \sqrt{n} \rightarrow \infty$ as $n \rightarrow \infty$.

For $t_{0}=t_{0}(n)$ we denote

$$
\underline{\tau}:=\lim \inf _{n \rightarrow \infty} \frac{t_{0}}{n}, \quad \bar{\tau}:=\lim \sup _{n \rightarrow \infty} \frac{t_{0}}{n} .
$$

By the assumption, $0<\underline{\tau} \leq \bar{\tau}<1$. In case that $0 \leq[n z] \leq t_{0}$, we have

$$
\frac{1}{\sqrt{n}}\left|\lambda_{n}(z)\right| \geq \frac{[n z]}{\sqrt{n}}\left|\delta_{0}\right|(1-\bar{\tau}) \text {. }
$$

Also in case that $t_{0}<[T z] \leq T$, we have

$$
\frac{1}{\sqrt{n}}\left|\lambda_{n}(z)\right| \geq \frac{n-[n z]}{\sqrt{n}}\left|\delta_{0}\right| \underline{\tau} .
$$

Thus

$$
\sup _{0 \leq z \leq 1} \frac{1}{\sqrt{n}}\left|\lambda_{n}(z)\right| \geq \max \left\{\sup _{0 \leq z \leq \frac{t_{0}}{n}} \frac{[n z]}{\sqrt{n}}\left|\delta_{0}\right|(1-\bar{\tau}), \sup _{\frac{t_{0}}{n}<z \leq 1} \frac{n-[n z]}{\sqrt{n}}\left|\delta_{0}\right| \underline{\tau}\right\} .
$$

Note that since $1-\bar{\tau}>0$ and $\underline{\tau}>0$, we have

$$
\lim _{n \rightarrow \infty} \max \left\{\sup _{0 \leq z \leq \frac{t_{0}}{n}} \frac{[n z]}{\sqrt{n}}\left|\delta_{0}\right|(1-\bar{\tau}), \sup _{\frac{t_{0}}{n}<z \leq 1} \frac{n-[n z]}{\sqrt{n}}\left|\delta_{0}\right| \underline{\underline{\tau}}\right\}=C \lim _{n \rightarrow \infty} \sqrt{n}\left|\delta_{0}\right|
$$

for some positive $C$. Since $\delta_{0}>0$ under the alternative hypothesis, the right-hand side is $\infty$. Thus the desired consistency result for $Q_{n}^{* M}$ is obtained. For other $R_{n}^{*}=Q_{n}^{* V}, P_{n}^{* M}$, or $P_{n}^{* V}$, similar arguments can be given to prove the consistencies, and here we omit the details because the discussions are almost the same except for $y_{t}$, replaced by $Y_{t}^{2}, \hat{a}_{t}$, or $\hat{b}_{t}$.

\section{Acknowledgement}

This study was supported by grants from the National Research Foundation of Korea (2016R1A2B400 8780, NRF-2015-1006133).

\section{References}

Baillie RT (1996). Long memory processes and fractional integration in econometrics, Journal of Econometrics, 73, 5-59.

Billingsley P (1999). Convergence of Probability Measures (2nd ed), Wiley, New York. 
Brown RL, Durbin J, and Evans JM (1975). Techniques for testing the constancy of regression relationships over time, Journal of the Royal Statistical Series B (Methodological), 37, 149-192.

Bühlmann P (2002). Bootstraps for time series, Statistical Science, 17, 52-72.

Corsi F (2004). A Simple Long Memory Model of Realized Volatility, Manuscript, University of Southern Switzerland, Lugano.

Corsi F (2009). A simple approximate long-memory model of realized volatility, Journal of Financial Econometrics, 7, 174-196.

Deng A and Perron P (2008). The limit distribution of the CUSUM of squares test under general mixing conditions, Econometric Theory, 24, 809-822.

Gonçalves S and de Jong R (2003). Consistency of the stationary bootstrap under weak moment conditions, Economics Letters, 81, 273-278.

Hwang E and Shin DW (2012). Strong consistency of the stationary bootstrap under $\psi$-weak dependence, Statistics and Probability Letters, 82, 488-495.

Hwang E and Shin DW (2013). A CUSUM test for a long memory heterogeneous autoregressive model, Economics Letters, 121, 379-383.

Hwang E and Shin DW (2014). Infinite-order, long-memory heterogeneous autoregressive models, Computational Statistics and Data Analysis, 76, 339-358.

Hwang E and Shin DW (2015). A CUSUMSQ test for structural breaks in error variance for a long memory heterogeneous autoregressive model, Statistics and Probability Letters, 99, 167-176.

Lee $\mathrm{O}$ (2014). The functional central limit theorem and structural change test for the HAR $(\infty)$ model, Economics Letters, 124, 370-373.

Parker C, Paparoditis E, and Politis DN (2006). Unit root testing via the stationary bootstrap, Journal of Econometrics, 133, 601-638.

Ploberger W and Krämer W (1986). On studentizing a test for structural change, Economics Letters, 20, 341-344.

Ploberger W and Krämer W (1990). The local power of the CUSUM and CUSUM of squares tests, Econometric Theory, 6, 335-347.

Ploberger W and Krämer W (1992). The CUSUM test with OLS residuals, Econometrica, 60, 271285.

Politis DN and Romano JP (1994). The stationary bootstrap, Journal of the American Statistical Association, 89, 1303-1313.

$\mathrm{Qu} \mathrm{Z}$ and Perron P (2007). Estimating and testing multiple structural changes in multivariate regressions, Econometrica, 75, 459-502.

Schwert GW (1989). Tests for unit roots: a Monte Carlo investigation, Journal of Business \& Economic Statistics, 7, 147-159.

Xu KL (2013). Powerful tests of structural changes in volatility, Journal of Econometrics, 173, 126142.

$\mathrm{Xu}$ KL (2015). Testing for structural change under non-stationary variances, Econometrics Journal, 18, 274-305. 